\title{
Is the role of trabeculectomy in glaucoma management changing?
}

\begin{abstract}
Background In recent years there have been significant developments in the diagnosis and treatment of glaucoma. We conducted a study to determine whether there has been an associated change in trabeculectomy rates in England over this period.

Methods Figures for the total number of trabeculectomies and cataract operations performed in England for each year from 1989/ 90 to $1999 / 2000$ were obtained from the Department of Health, Hospital Episode Statistics Division.

Results The available data show that the number of trabeculectomies and cataract operations increased year on year from 1990/91 until 1995/96. Since then, the number of cataract procedures has continued to rise dramatically, whereas the number of trabeculectomies has fallen progressively by $7 \%, 23 \%, 37 \%$ and $53 \%$ in the last 4 years. Conclusion The perceived need for surgical intervention would appear to have been delayed or prevented. We postulate that several factors underlie this trend and that there are a number of implications regarding the future management of glaucoma patients.
\end{abstract}

\section{Key words Glaucoma, Trabeculectomy}

Trabeculectomy was first described by Cairns in $1968,{ }^{1}$ and since then it has remained by far the most commonly performed glaucoma filtration procedure. There has been debate regarding the timing of trabeculectomy in glaucoma patients. Advocates of primary surgery report success rates of approximately $90 \%,{ }^{2,3}$ and an additional benefit of this approach may be the avoidance of visual field loss whilst attempting to control glaucoma medically. However, in most cases surgery is considered only where maximal medical treatment has failed to halt disease progression. ${ }^{4}$ Amongst patients who have received long-term medical treatment prior to surgery, the success rates of trabeculectomies tend to be lower, ranging from $45 \%$ to $93 \%{ }^{5}$ Over the past decade there have been significant developments in the medical treatment of
K.W. WHITTAKER, J.T. GILLOW,

I.A. CUNLIFFE glaucoma and it has been suggested that patient management strategies are likely to change with medical therapy being increasingly preferred in lieu of surgical intervention. ${ }^{6}$ Developments in the diagnosis and monitoring of glaucoma may also affect the relative use of medical and surgical management.

In the West Midlands we recently detected a fall in trabeculectomy rates. ${ }^{7}$ We are aware that the National Survey of Trabeculectomy showed considerable variation in the approach to glaucoma management across the country. ${ }^{4} \mathrm{We}$, therefore, conducted this study to determine whether our observation was a regional issue or part of a nationwide trend.

\section{Method}

The Department of Health, Hospital Episode Statistics Division, was asked to provide data on Finished Consultant Episodes (FCEs) in NHS hospitals in England. A FCE is defined as a period of patient care under one consultant within one healthcare provider. Specifically, figures were requested for the number of trabeculectomies (operation code C60.1) and cataract operations (operation codes C71.1, C71.2, C71.3, C71.8 and C71.9) performed for each of the past 11 years. The figures for cataract surgery were obtained for use as a marker of the general ophthalmic surgical workload against which trabeculectomy rates could be compared.

In addition, the Department of Health also provided a national population estimate for 1992 and 1997.

\section{Results}

The number of trabeculectomy and cataract procedures performed in NHS hospitals in England between 1989/90 and 1999/2000 is shown in Table 1 . The data for $1989 / 90$ to $1997 / 98$ are complete and the figures are final. The data for 1998/99 and 1999/2000 are 'ungrossed', i.e. the figures are provisional pending adjustments for shortfalls owing to missing or invalid data. Analysis by the Department of Health has shown the figures for these last 2 years are likely to be very accurate.

\author{
K.W. Whittaker \\ I.A. Cunliffe \\ Birmingham and Midland \\ Eye Centre \\ City Hospital NHS Trust \\ Birmingham B18 7QH, UK \\ J.T. Gillow \\ Department of \\ Ophthalmology \\ North Staffordshire Hospital \\ Stoke-on-Trent ST4 7LN, UK \\ Mr K.W. Whittaker \\ Birmingham and Midland \\ Eye Centre \\ City Hospital NHS Trust \\ Birmingham B18 7QH, UK
}

Received: 4 August 2000 Accepted in revised form: 6 April 2001 


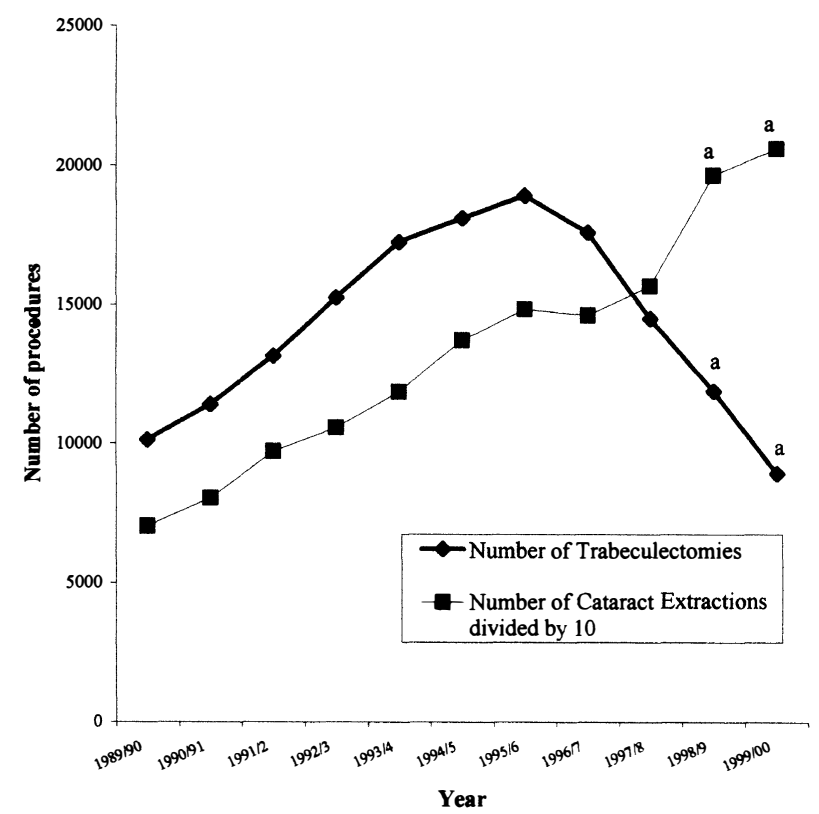

Fig. 1. Number of trabeculectomies and cataract extractions performed 1989/90 to 1999/2000. ${ }^{a}$ Predicted final ('grossed') figures.

It estimates that, relative to the 'ungrossed' figures, the final number of procedures will be approximately $2 \%$ higher for 1998/9 and 3\% higher for 1999/2000. The predicted final figures for these years, based on these percentage 'uplifts', are shown in parentheses in Table 1.

The data are displayed graphically in Fig. 1. This shows that the number of trabeculectomies and cataract operations has increased year on year from 1989/90 until $1995 / 96$. Since then, the number of cataract procedures has continued to rise, whereas the number of trabeculectomies has fallen progressively by $7 \%, 23 \%$, $37 \%$ and $53 \%$ in the last 4 years (using the predicted final figures for the last 2 years). A straight-line fit (using Least Squares Regression Analysis) was obtained for the figures for cataract surgery from 1989/90 to $1999 / 2000$ (positive slope, $p<0.05$ ). Statistical analysis of the trabeculectomy data showed a significant straight-line fit with a positive slope for the 1989 /90 to 1995 /96 data, and a negative slope for $1995 / 1996$ to $1999 / 2000$ $(p<0.05)$.

The national population for residents over 65 years of age has increased by 1.6\% from 1992 to 1997.

\section{Discussion}

The National Survey of Trabeculectomy confirmed that trabeculectomy was practised by the majority of consultant ophthalmologists in the UK and estimated that 21600 trabeculectomies were performed on $4 \%$ of glaucoma patients in $1995 .{ }^{4}$ This figure is comparable with the Department of Health statistics shown in Table 1. However, since this time there appears to have been a reduction in the perceived need for surgical intervention in glaucoma patients in England. The available data show a progressive fall in the number of trabeculectomies performed since 1995/6, with an overall reduction of more than $50 \%$. This has occurred in spite of a greater than $30 \%$ increase in the general ophthalmic surgical workload, measured in terms of the number of cataract operations undertaken in the same period. The downward trend in trabeculectomy numbers has also occurred in spite of an increase in the national population aged 65 or over, in whom the prevalence of glaucoma and the requirement for surgical intervention is highest. ${ }^{4}$

We acknowledge that the figures for 1998/99 and $1999 / 2000$ are provisional. However, analysis by the Department of Health suggests the impact from any shortfall in this data is likely to be minimal. Furthermore, the downward trend in trabeculectomy figures resulting from the predicted 'grossed' data is in keeping with the reduction in numbers since 1995/6-1997/8, for which the final figures are available. Similarly, the predicted final figures for cataract surgery continue the linear rise noted in previous years.

Several plausible explanations for this apparent change in glaucoma management strategy should be considered, including:

1. Glaucoma referral patterns may have changed. Patients may be presenting earlier to the Hospital Eye Service with less advanced disease, which is more effectively managed medically. Assuming the prevalence of glaucoma has remained stable, it seems unlikely that the number of glaucoma outpatient referrals has fallen. The average interval between optometric sight tests for people over the age of 60 years was similar in the early 1980s and early $1990 \mathrm{~s}^{8}$ and there seems no reason for this pattern to have altered.

2. The introduction of newer-generation ocular hypotensive medications may have delayed or eliminated the need for surgery in some patients. United Kingdom licences were granted for dorzolamide in 1995, for latanoprost and brimonidine in 1997 and for dorzolamide-timolol in 1998. Collectively, these now make up over one-

Table 1. Trabuculectomy and cataract extraction figures $1989 / 90$ to $1999 / 2000$

\begin{tabular}{lcccccccccccc}
\hline & $1989 / 90$ & $1990 / 1$ & $1991 / 92$ & $1992 / 3$ & $1993 / 4$ & $1994 / 5$ & $1995 / 6$ & $1996 / 7$ & $1997 / 8$ & $1998 / 9$ & $1999 / 00$ \\
\hline Trabeculectomies & 10115 & 11404 & 13158 & 15257 & 17249 & 18112 & 18928 & 17595 & 14507 & 11882 & 8914 \\
& & & & & & & & & \\
$(12120)^{a}$ & $(9181)^{a}$ \\
Cataract extractions & 70197 & 80323 & 97026 & 105587 & 118550 & 137213 & 148427 & 146173 & 156778 & 196584 & 206057 \\
\hline
\end{tabular}

Figures for 1998/99 and 1999/2000 are provisional ('ungrossed').

${ }^{a}$ Predicted final ('grossed') figures are given in parentheses. 
third of all glaucoma prescriptions, the total number of which has increased year on year over the past 5 years. ${ }^{9}$

3. The use of automated perimetry has probably become more widespread. With the greater accuracy and sensitivity afforded in comparison with manual techniques, it is possible that glaucoma is being detected earlier with less need for surgical intervention.

4. Advances in surgical technique and awareness of the importance of careful post-operative management may have reduced the need for repeat procedures. Antimetabolites have been shown to improve the success rate of trabeculectomy in highrisk cases. ${ }^{10,11}$

5. The fashion for primary trabeculectomies may be fading, leading to a reduction in the total figure for the number of trabeculectomies performed. However, glaucoma filtration surgery may still be just as common as a last line of defence when medical treatment fails.

6. The number of trabeculectomies performed in private hospitals may be increasing. The data available refer only to procedures carried out in NHS hospitals. However, any increase is likely to be relatively small, causing little impact on the observed trend.

Clearly, patients will benefit from the recent advances in earlier glaucoma detection, and medical and surgical management. However, ophthalmologists should be aware that the development of a trend away from surgical intervention may have important implications for the future management of glaucoma patients and the allocation of resources and training. We anticipate a potential impact upon several issues.

Firstly, with the increased range of therapeutic options now available, ophthalmologists must be aware of a potential for greater risk of disease progression to visually significant levels whilst trying to control intraocular pressure. It has been estimated that visually significant visual field loss occurs in almost one third of patients whilst attempting to control intraocular pressure medically before listing for surgery. ${ }^{3}$

Secondly, subconjunctival fibroblasts play a critical role in scarring and treatment failure in glaucoma filtration surgery. Long-term prior treatment with adrenaline and miotics has been shown to be associated with increased numbers of inflammatory cells and fibroblasts in the conjunctival and episcleral tissues and higher failure rates for trabeculectomy. ${ }^{12,13}$ There is contradictory evidence for there being a similar effect with the newer ocular hypotensive agents. When applied to rabbit eyes for 10 weeks, latanoprost and brimonidine were shown to increase the number of proliferating subconjunctival fibroblasts. ${ }^{14}$ Conversely, histopathological examination of conjunctival biopsies in 5 patients who received latanoprost monotherapy over a 12 month period showed (in one abstract) no alteration to the conjunctival epithelium, goblet cell population or mucus quality, although a focal increase in inflammatory cells or vascular congestion was observed in some patients. ${ }^{15}$ Larger studies are required to determine the effect of prolonged use of the newer agents on trabeculectomy 'failure' rates, and whether there will be a greater need for the use of adjunctive surgical agents.

Thirdly, further consideration may need to be given to the surgical role of future non-glaucoma specialists. A recent amendment of the 'Curriculum of Higher Specialist Training in Ophthalmology' by the Royal College Training Committee includes a 'reduction in essential glaucoma surgical experience from a minimum of 30 intraocular surgical cases and 20 anterior segment lasers to a minimum of 30 surgical and laser procedures for glaucoma. ${ }^{16}$ This may be a reflection of reduced exposure to surgical management of glaucoma by current Specialist Registrars.

Fourthly, an increasing trend towards medical treatment may affect the economic evaluation of glaucoma care. In the early 1990s, early surgery was demonstrated to be no more expensive than conventional management. ${ }^{17}$ It has since been shown that patient management costs are directly related to changes in medical treatment, ${ }^{18}$ the number of which may increase with the greater range of options now available.

In conclusion, this study highlights an apparent reduction in trabeculectomy rates. Given the implications discussed, we suggest there is a need for a national re-audit of trabeculectomy in the next few years. In addition, consideration should be given to the training requirements of Specialist Registrars regarding glaucoma management and to the economic consequences of this trend.

\section{References}

1. Cairns JE. Trabeculectomy: preliminary report of a new method. Am J Ophthalmol 1968;66:673-79.

2. Jay JL, Murray SB. Early trabeculectomy versus conventional management in primary open angle glaucoma. $\mathrm{Br} \mathrm{J}$ Ophthalmol 1988;72:881-9.

3. Migdal C, Hitchings RA, Gregory W. Primary treatment trial of visual fields. Eye 1993;114:737-41.

4. Edmunds B, Thompson JR, Salmon JF, Wormald RP. The National Survey of Trabeculectomy. I. Sample and methods. Eye 1999;13:524-30.

5. Broadway DC, Grierson I, O'Brien C, Hitchings RA. Adverse effects of topical anti-glaucomatous medication regimens. II. Effects on the outcome of filtration surgery. Arch Ophthalmol 1994;112:1446-54.

6 . Gualano J. The place and role of evidence-based medicine in the treatment of glaucoma. Eye News 1998;5(2):15-20.

7. West Midlands NHS Executive, Hospital Episodes Statistics Database 1991-7.

8. Vernon SA. The changing pattern of glaucoma referrals by optometrists. Eye $1998 ; 12: 854-7$.

9. DIN-LINK Market Research.

10. Flourouracil Filtering Surgery Study Group. Three-year follow-up of the Flourouracil Filtering Surgery Study. Am J Ophthalmol 1993;115:82-92.

11. Palmer SS. Mitomycin as adjunct chemotherapy with trabeculectomy. Ophthalmology 1991;98:317-21. 
12. Baudouin C. Mechanisms of failure in glaucoma filtration surgery: a consequence of antiglaucomatous drugs? J Clin Pharmacol Res 1996;16(1):29-41.

13. Sherwood MB, Grierson I, Millar L, Hitchings RA. Long-term morphological effects of anti-glaucoma drugs on the conjunctiva and Tenon's capsule in glaucomatous patients. Ophthalmology 1989;96:327-35.

14. Lark KK, Pasha AS, Yan X, Edward DP. The effect of latanoprost and brimonidine on rabbit subconjunctival fibroblasts. J Glaucoma 1999;8:72-6.

15. Petounis A. Histopathological study of conjunctiva following long-term lanatoprost treatment [Abstract]. Meeting of the European Glaucoma Society, London 2000.
16. Curriculum of Higher Specialist Training in Ophthalmology, updated version November 2000. London: Royal College of Ophthalmologists, 2000.

17. Ainsworth JR, Jay JL. Cost analysis of early trabeculectomy versus conventional management in primary open angle glaucoma. Eye 1991;5:322-8.

18. Kobelt-Nguyen G, Gerdtham UG, Alm A. Costs of treating primary open angle glaucoma and ocular hypertension: a retrospective, observational two-year chart review of newly diagnosed patients in Sweden and the United States. J Glaucoma 1998;7:95-104. 